\title{
Proposed National Large Solar Telescope
}

\author{
Jagdev Singh \\ Indian Institute of Astrophysics, Bangalore 560 034, India. \\ e-mail: jsingh@iiap.res.in
}

\begin{abstract}
Sun's atmosphere is an ideal place to study and test many magnetohydrodynamic (MHD) processes controlling turbulent plasma. We wish to resolve some of the finest solar features (which remain unresolved presently) and study their dynamics. Indian Institute of Astrophysics has proposed to design, fabricate and install a 2-meter class solar telescope at a suitable site in India to resolve features on the Sun of the size of about 0.1 arcsec. The focal plane instruments will include a high resolution polarimeteric package to measure polarization with an accuracy of 0.01 per cent; a high spectral resolution spectrograph to obtain spectra in 5 widely separated absorption lines simultaneously and high spatial resolution narrow band imagers in various lines. The Himalayan region appears to be a good choice keeping in view the prevailing dry and clear weather conditions. We have started detailed analysis of the weather conditions in the area and at some other locations in India. The site characterization will be done using the Sun-photometer, S-DIMM and SHABAR techniques to determine the seeing conditions.
\end{abstract}

Key words. Solar telescope-adaptive optics—spectropolarimetrylocation.

\section{Introduction}

The Sun offers an excellent laboratory to address the key processes that take place in highly magnetized plasma that govern the many astrophysical phenomena and thus provides a stepping stone to infer physical parameters in other more complex stellar systems and of the universe at large. All the observatories in India (Kodaikanal, Nainital and Udaipur) have made significant contribution to solar physics. Kodaikanal observatory has obtained photoheliograms and $\mathrm{Ca}-\mathrm{K}$ and $\mathrm{H}-\alpha$ spectroheliograms of the Sun over a period of about 100 years and formed unique datasets to study the long period variations in various physical phenomena on the Sun because of uniformity in the data (Singh \& Bappu 1981; Singh \& Prabhu 1985; Sivaraman et al. 1999; Makarov et al. 2001). Apart from the study of long period variations, one needs to investigate very small scale structures such as flux tubes that play an important role in all the activity and physical processes on the Sun. One meter Swedish vacuum solar telescope at La Palma (a beautiful sight with very stable and transparent sky conditions), Canary Islands which is being operated for the last 5 years by the Royal Academy of Sciences, 
Sweden has yielded great details about the solar surface features (Scharmer et al. 2002) of the order of $0.3 \mathrm{arcsec}$. The $65-\mathrm{cm}$ telescope at Big Bear Solar Observatory has been used to take images and make polarization measurements in $1565 \mathrm{~nm}$ line recently (Cao et al. 2006a, 2006b). They could achieve a spatial resolution of $0.3 \mathrm{arcsec}$ using adaptive optics. Kiepenheuer Institute of Solar Physics, Germany is planning Gregorian, on-axis, Alt-Azimuth, $1.5 \mathrm{~m}$ open telescope to be installed at Izana. Big Bear Solar Observatory is planning to fabricate and install $1.6 \mathrm{~m}$ off-axis in place of $65 \mathrm{~cm}$ existing telescope. The upgraded facility will be used for the existing programs and magnetic field measurements with better spatial resolution and photometric accuracy.

A large solar telescope known as Advanced Technology Solar Telescope (ATST) of 4-meter size, capable of taking diffraction limited images of the Sun (spatial resolution of $0.03 \mathrm{arcsec}$ ) and make observations in the infrared wavelength region, has been proposed by the National Solar Observatory in collaboration with many organizations (ATST report 2000). The proposed telescope will be capable of observing the solar corona also. It is likely to come up in the near future. There are many scientific objectives of the telescope:

- How are the highly intermittent magnetic fields observed at the solar surface generated by dynamo processes and how are they dissipated?

- What magnetic configurations and evolutionary paths lead to flares and coronal mass ejections?

- What mechanisms are responsible for variations in the spectral and total irradiance of the Sun and solar-type stars?

- Progress in answering these critical questions requires a study of the interaction of the magnetic field and convection with a resolution sufficient to observe scales fundamental to these processes. Recently a 50-cm telescope known as Hinode has been launched in space by a Japanese group in collaboration with USA and UK scientists and has obtained diffraction limited images with a spatial resolution of 0.2 arcsec in G-band, $\mathrm{Ca}-\mathrm{H}$ line and have made magnetic field measurements (Hinode 2006). The images indicate that flux tubes still need to be resolved by making observations with higher spatial resolution. ATST is planning to achieve this and has many other objectives that require a large size aperture of about $4 \mathrm{~m}$. We, in India plan to have limited scientific objectives such as to resolve the flux tubes, make magnetic field measurements with required accuracy and some other objectives listed in Table 2 for which a $2 \mathrm{~m}$ class telescope seems to be sufficient.

It is well known that existing observational facilities for solar research in India, the latest being Solar Tower Telescope installed in the year of 1960, are grossly inadequate for high resolution observations of the Sun. Absence of a large and versatile telescope, which can facilitate simultaneous measurements of the solar atmospheric parameters and of the vector magnetic fields with high accuracy, has been a serious handicap for the solar astronomers. Efforts have been made for the past 20 years or so to have a good and large solar telescope, equipped with state-of-the-art focal plane instruments using the present day technology but without success. Recently Udaipur Solar Observatory has succeeded in designing a moderate size telescope and placed an order with a company (Venkatakrishnan 2006). There is therefore, an imperative need for a state-of-the-art solar observational facility, comparable to the best in other countries. A 2-meter class 
large solar telescope is hence being proposed as a national facility for implementation during the 11th five year plan.

\section{Scientific objectives}

The proposed telescope will address the fundamental questions about the nature of solar magnetism, will aim to resolve flux tubes and measure their strength; address the development of magnetic fields on the Sun which are responsible for almost all the observable phenomena on the Sun such as solar dynamo, solar cycle and solar variability that determine and control the space weather. The other scientific objectives are: (i) MHD waves by resolving small structures and determining periods of oscillations which may be responsible to transport the energy to the upper atmosphere of the Sun; (ii) Dynamic evolution of small scale structures by making high cadence observations; (iii) Evolution of active regions and their role in triggering solar flare, prominences, filament eruptions, CMEs, etc.; (iv) Thermodynamics of the chromosphere by making the observations in the infrared wavelengths; (v) Weak and turbulent magnetic field measurements using Hanle effect which are as important as the strong magnetic fields and have now become possible to be measured because of the development in technology. All these objectives will be achieved by making observations with high spatial resolution using adaptive optics, high spectral resolution, high temporal resolutions, multi-wave length capability of imaging and spectroscopy focal plane instruments, high photon flux and sensitivity of the detectors and using the infrared part of the spectrum for observations. Table 1 gives a summary of the scientific objectives.

\section{Telescope specifications}

Keeping in view the scientific objectives and technical requirements of observations, it has been proposed to have a $2 \mathrm{~m}$ class telescope of Gregorian format. Direct light from the Sun falls on the primary mirror M1 of aperture $2 \mathrm{~m}$ and of a focal ratio of $\mathrm{f} / 2.0$ which forms a solar image of about $36 \mathrm{~mm}$ size at the prime focus F1. In the process of forming the solar image, the primary mirror concentrates nearly $3 \mathrm{~kW}$ of heat at the focus. A heat trap (not shown in Fig. 1) with an efficient circulating coolant placed close to F1 takes away more than $98 \%$ of the heat by directing most of the solar radiations out of the telescope to the atmosphere. A field stop with a hole of about $6 \mathrm{~mm}$ diameter allows only a small portion of the solar image (about $300 \mathrm{arcsec}$ ) to pass through further to the secondary mirror M2. This helps to reduce the heat further within the telescope. The secondary mirror M2 magnifies the $300 \mathrm{arcsec}$ of the Sun's image and brings it to the secondary focus F2. The beam is allowed to proceed further to the tertiary mirror M3 of the Gregorian system which magnifies the image at the secondary focus and brings the 300 arcsec image of the Sun to the final focus point F3 via a chain of flat mirrors M4, M5, M6, M7, and M8 into the laboratory at the lower level as shown in Fig. 1. M6, M7, and M8 form a de-rotator that compensates for the rotation of the field of view. Mirror M9, serves as the tip tilt mirror (active optics mirror) and M10 is the deformable mirror DM of the adaptive optics. The mirror M11 feeds light to the chosen focal plane instruments for various observational programs through set of beam splitters and mirrors. The primary image formed by the mirror M1 will have aberrations (coma and astigmatism). These are 
Table 1. Summary of science objectives and matching instrumentation.

\begin{tabular}{|c|c|c|c|}
\hline \multicolumn{4}{|c|}{ Summary of science objectives } \\
\hline $\begin{array}{l}\text { Physical pro- } \\
\text { cess/ region to be } \\
\text { observed }\end{array}$ & $\begin{array}{l}\text { Physical quantity to } \\
\text { be measured }\end{array}$ & $\begin{array}{l}\text { Telescope/instrument } \\
\text { requirement }\end{array}$ & $\begin{array}{l}\text { Instruments } \\
\text { proposed }\end{array}$ \\
\hline \multirow[t]{4}{*}{$\begin{array}{l}\text { MHD waves and } \\
\text { oscillations }\end{array}$} & $\begin{array}{l}\text { Intensity variation of } \\
1 \% \text { or less }\end{array}$ & $\begin{array}{l}\text { High photon flux need- } \\
\text { ing aperture of } 2 \text { meter } \\
\text { and above }\end{array}$ & $\begin{array}{l}\text { Narrow band } \\
\text { filters }\end{array}$ \\
\hline & $\begin{array}{l}\text { Velocities of } \\
\sim 200 \mathrm{~m} / \mathrm{sec} \text { or less }\end{array}$ & $\begin{array}{l}\text { Spectral resolution of a } \\
\text { few } m \AA\end{array}$ & $\begin{array}{l}\text { High resolution } \\
\text { spectrograph }\end{array}$ \\
\hline & $\begin{array}{l}\text { Magnetic field mea- } \\
\text { surements }\end{array}$ & $\begin{array}{l}\text { Polarization accuracy: } \\
10^{-4} \text { or better }\end{array}$ & $\begin{array}{l}\text { Spectropolarimeter } \\
\text { with spatial } \\
\text { information }\end{array}$ \\
\hline & $\begin{array}{l}\text { Properties of oscilla- } \\
\text { tions }\end{array}$ & High time cadence & $\begin{array}{l}\text { High } \\
\text { cameras }\end{array}$ \\
\hline $\begin{array}{l}\text { Different heights } \\
\text { in solar atmo- } \\
\text { sphere }\end{array}$ & $\begin{array}{l}\text { Diagnostic parame- } \\
\text { ters covering many } \\
\text { spectral lines }\end{array}$ & $\begin{array}{l}\text { Both visible and IR } \\
\text { capabilities }\end{array}$ & $\begin{array}{l}\text { Ca II K, H } \alpha, \mathrm{G} \\
\text { band, He I } 1083 \mathrm{~nm} \text {, } \\
\text { Fe I } 1.56 \text { microns } \\
\text { filters }\end{array}$ \\
\hline $\begin{array}{l}\text { Active region evo- } \\
\text { lution }\end{array}$ & $\begin{array}{l}\text { Velocity and mag- } \\
\text { netic fields }\end{array}$ & $\begin{array}{l}\text { High time cadence } \\
\text { large field of view of } \\
300 \text { arcsec }\end{array}$ & $\begin{array}{l}\text { Spectropolarimeter } \\
\text { with spatial } \\
\text { information }\end{array}$ \\
\hline Hanle effect & $\begin{array}{l}\text { Magnetic field } \\
\sim 5-10 \mathrm{G}\end{array}$ & $\begin{array}{l}\text { Polarization accuracy } \\
10^{-4}\end{array}$ & Spectropolarimeter \\
\hline
\end{tabular}

properly corrected by mirrors M2 and M3 to produce the final aberration-free image of the 300 arcsec of the Sun at the final focus. In addition, these two mirrors will be mounted on hexapods to enable fine tuning of the alignment of the telescope system. The f-ratio of the telescope system will be designed to be $\mathrm{f} / 40$ so that the final magnified image of the Sun will have a scale of $\sim 2.5 \mathrm{arcsec} / \mathrm{mm}$ at the final focal plane.

One of the major focal plane instruments will be a spectropolarimeter package to measure the magnetic fields with a high degree of accuracy and high spatial resolution. The spectropolarimeter will use the new technology of Dense Wave Division Multiplexing (DWDM) filters and Fiber bundles to convert two dimensional solar images into slit images to perform spectroscopy. This will avoid scanning of the image to measure the magnetic fields and thus permit study of the temporal variations in the magnetic fields before, during and after the solar flares. This will help us to understand the processes involved in the triggering of flares. A high spectral resolution spectrograph capable of taking spectra in 5 widely separated absorption lines simultaneously and a facility to take high spatial resolution narrow band images of the Sun in many photospheric and chromospheric lines to resolve structures of about $50 \mathrm{~km}$ size. The optical layout of the telescope is shown in Fig. 1 and the specifications are indicated in Table 2. The narrow band filters will include Ca-K, H- $\alpha, \mathrm{CN}-$ band, G-band and UBF with $0.02 \mathrm{~nm}$ pass band. The telescope will employ active and adaptive 


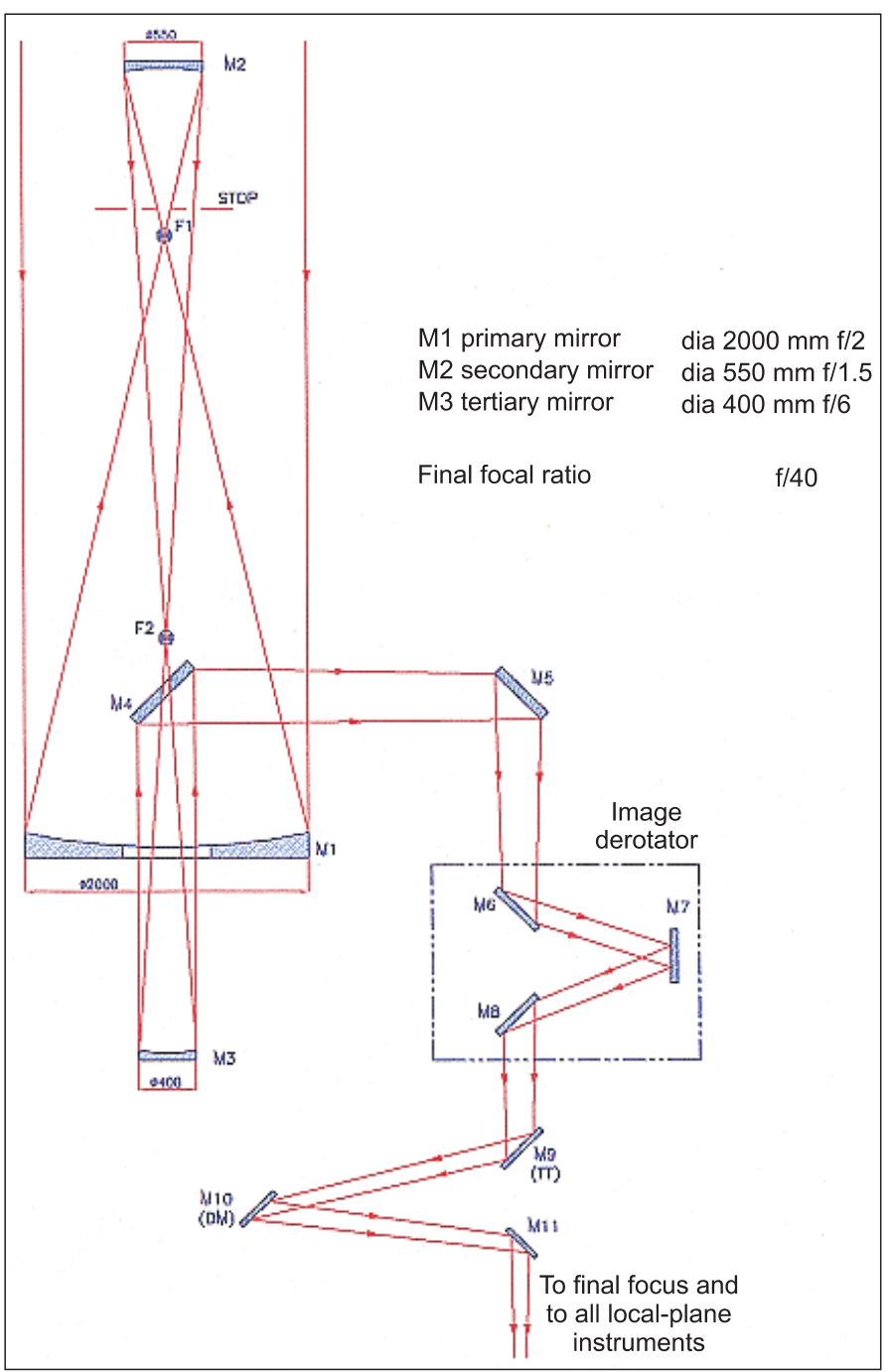

Figure 1. Optical layout of the proposed $2 \mathrm{~m}$ solar telescope.

optics to stabilize the image and reduce the effect of variations in the atmospheric conditions.

\section{Location and site survey}

The location of the telescope needs to provide a large number of clear hours for making observations with very good seeing and transparency. To make observations in the infrared wavelengths for high accuracy of magnetic field and velocity measurements, water vapours in the air need to be very less. Himalayan regions appear to provide such atmospheric conditions. A $2 \mathrm{~m}$ telescope has already been established at Hanle to perform astronomical observations during night-time. The site survey done at various 
Table 2. Summary of telescope features.

\begin{tabular}{ll}
\hline Aperture (primary mirror M1) & 2 meters \\
Focal length & 4 meters \\
Optical configuration & 3 mirror, Gregorian on - axis \\
Field of view & 300 arcsec \\
Final focal ratio of the system & $\mathrm{f} / 40$ \\
Image scale & $2.58 \mathrm{arcsec} \mathrm{mm}^{-1}$ \\
Optical quality & $<0.1 \mathrm{arcsec}$ over the field of view \\
Wavelength of operation & $380 \mathrm{~nm}$ to $1000 \mathrm{~nm}$ (upto 2500) \\
Polarization accuracy & 1 part in 10,000 \\
Active and adaptive optics & To realize near diffraction limited performance \\
Spatial resolution & $<0.1$ arcsec
\end{tabular}

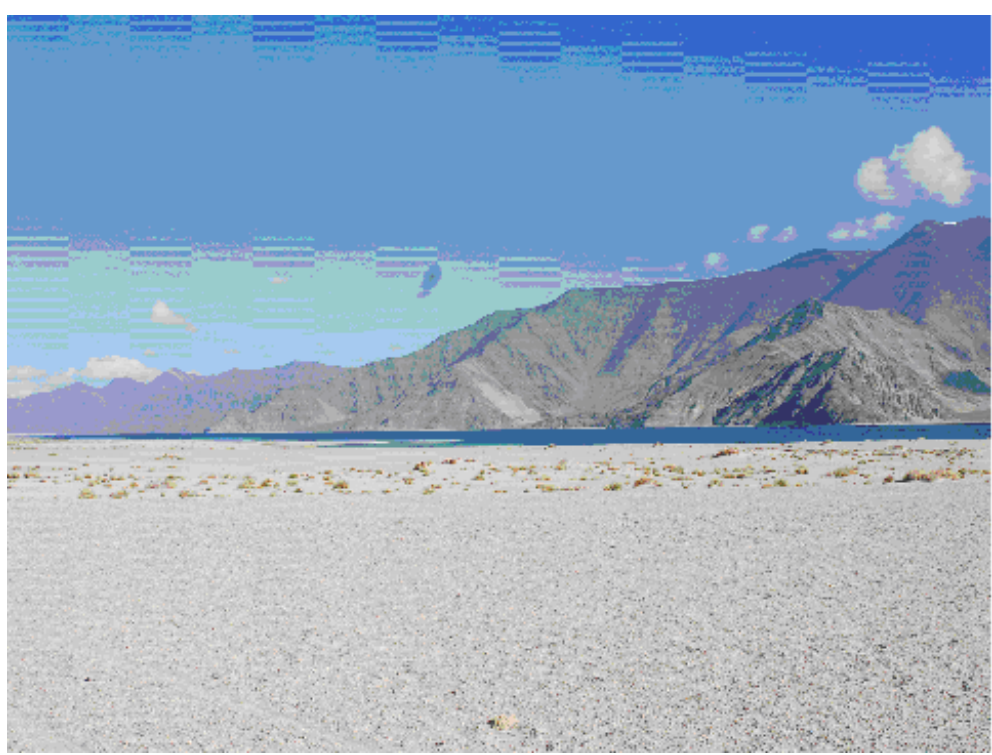

Figure 2. Part of the Pangong lake in the Himalayan region.

places has indicated that the lake sites provide better seeing conditions such as that at Big Bear Solar Observatory. We, therefore, have planned to determine the atmospheric conditions during daytime at Hanle (existing observatory), Pangong in the Himalayan region (a big lake site, Fig. 2) and Devasthal near Nainital Observatory). We have already collected the weather data at Hanle and at Devasthal. We have started to determine the weather conditions at Pangong as shown in Fig. 3. We plan to measure the seeing conditions at these places using S-DIMM and SHABAR techniques. We have already procured one such instrument from National Solar Observatory, USA which we expect to install at Hanle during the period of June 2007. We plan to fabricate such systems to measure the seeing conditions at other places mentioned above and at some other places chosen later. 


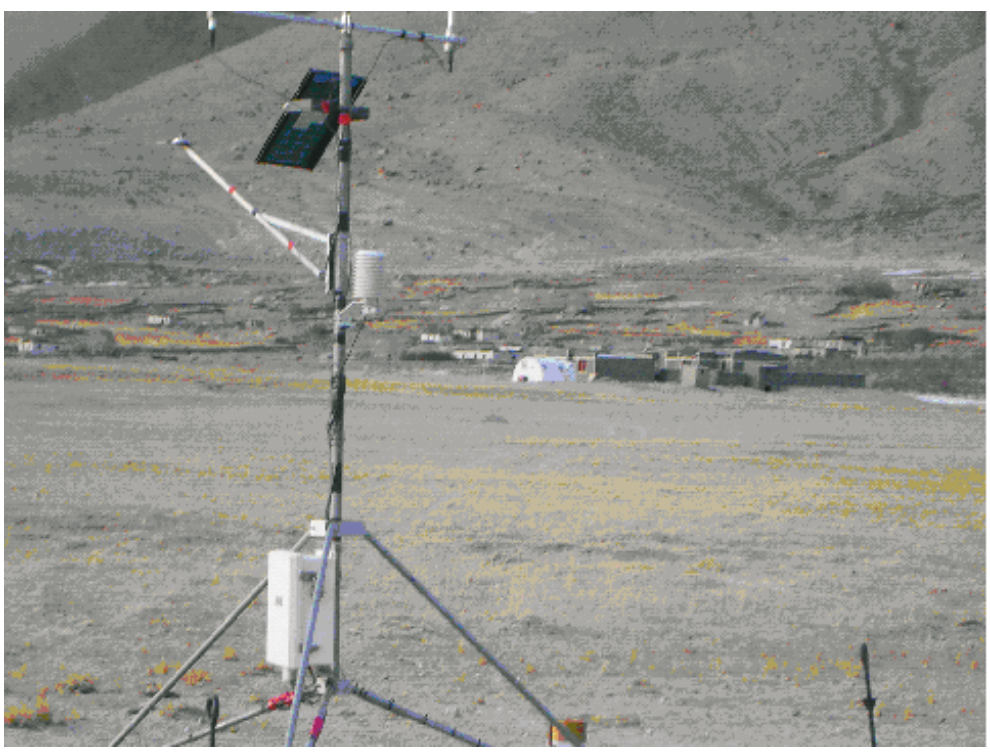

Figure 3. Weather station at Pangong to measure solar radiations, temperature, wind speed, etc.

\section{Acknowledgements}

This is based on the project report prepared by the members of solar group at Indian Institute of Astrophysics and many others. The weather data at Hanle was collected by the members of IIA and at Devasthal by members of ARIES.

\section{References}

Advanced Technology Solar Telescope Design and Development Phase (ATST) 2000, Report submitted by NSO, to National Science Foundation.

Cao, W., Jing, J., Ma, J., Xu, Y., Wang, H., Goode, P. R. 2006a, Pub. Astro. Soc. Pac., 118, 838. Cao, W., Hartkorn, K., Ma, J., Xu, Y., Spirock, T., Wang, H., Goode, P. R. 2006b, Solar Phys., 238, 207.

Hinode satellite (www. science.nasa.gov/headlines/y2006/02nov_firstlight.htm).

Makarov, V. I., Tlatov, A. G., Sivaraman, K. R. 2001, Solar Phys., 202, 11.

Scharmer, G. B., Gudiksen, B. V., Kiselman, D., Löfdahl, M. G., Rouppe van der, V., Luc, H. M. 2002, Nature, 420, 151.

Singh, J., Bappu, M. K. V. 1981, Solar Phys., 71, 161.

Singh, J., Prabhu, T. P. 1985, Solar Phys., 97, 203.

Sivaraman, K. R., Gupta, S. S., Howard, R. F. 1999, Solar Phys., 189, 69.

Venkatakrishnan, P. 2006, Private communication. 